\title{
Wetting of a Chemically Heterogeneous Surface
}

\author{
Laura J. Douglas Frink \\ Materials Simulation Science Department \\ Sandia National Laboratories \\ Albuquerque, New Mexico 87185-1111 \\ Andrew G. Salinger \\ Parallel Computational Sciences Department \\ Sandia National Laboratories \\ Albuquerque, New Mexico 87185-1111 \\ (October 7, 1998)
}

Theories for inhomogeneous fluids have focused in recent years on wetting, capillary condensation, and solvation forces for model systems where the surface(s) is(are) smooth homogeneous parallel plates, cylinders, or spherical drops. Unfortunately natural systems are more likely to be heterogeneous both in surface shape and surface chemistry. In this paper we discuss the consequences of chemical heterogeneity on wetting. Specifically, a 2-dimensional implementation of a nonlocal density functional theory is solved for a striped surface model. Both the strength and range of the heterogeneity are varied. Contact angles are calculated, and phase transitions (both the wetting transition and a local layering transition) are located. The wetting properties of the surface are shown to be strongly dependent on the nature of the surface heterogeneity. In addition highly ordered nanoscopic phases are found, and the operational limits for formation of ordered or crystalline phases of nanoscopic extent are discussed.

submitted to J.Chem. Phys. 10-8-98

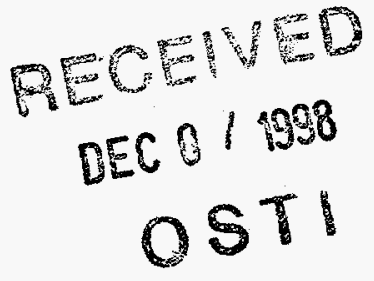




\section{DISCLAIMER}

This report was prepared as an account of work sponsored by an agency of the United States Government. Neither the United States Government nor any agency thereof, nor any of their employees, make any warranty, express or implied, or assumes any legal liability or responsibility for the accuracy, completeness, or usefulness of any information, apparatus, product, or process disclosed, or represents that its use would not infringe privately owned rights. Reference herein to any specific commercial product, process, or service by trade name, trademark, manufacturer, or otherwise does not necessarily constitute or imply its endorsement, recommendation, or favoring by the United States Government or any agency thereof. The views and opinions of authors expressed herein do not necessarily state or reflect those of the United States Government or any agency thereof. 


\section{DISCLAIMER}

Portions of this document may be illegible in electronic image products. Images are produced from the best available original document. 


\section{INTRODUCTION}

Tailoring materials at submicron lethgth scales is becoming increasingly important in applications ranging from microelectronics to micromachines to drug design. In general surfaces, gels, or macromolecules may be tailored for their shape [1], porosity [2], surface roughness [3], or chemistry [4]. In any case, it is important to be able to predict the effects of heterogeneities on the macroscopic properties of the material of interest. In this paper we focus on the effects of surface chemical heterogeneity on wetting.

In addition to those systems specifically designed for some type of chemical heterogeneity, many naturally occurring systems owe their unique functionality to similar heterogeneities on the molecular (nanoscopic) length scale. Amphiphilic molecules with polar (hydrophilic) head groups and nonpolar (hydrophobic) hydrocarbon tails can form micelles and other complex assemblies. These assemblies arise from the interaction of the amphiphile with the solvent (typically water). More specifically, the polar head groups arrange to form an interface that separates the hydrocarbon tails from the solvent [5-7]. Proteins are another example where both hydrophilic and hydrophobic amino acids appear on one macromolecule. The pattern and strength of interactions between amino acids is assumed to be the basis for protein folding [8-10].

In both macroscopic $[11,12]$ and statistical mechanical studies [13-15] of fluid-solid interfaces, the attraction of a fluid for a surface is often quantified by a contact angle, $\theta$. When $\theta=180^{\circ}$, the fluid-surface interaction is nonwetting, and liquid will be displaced from such a surface by an intervening vapor film. When $\theta=0^{\circ}$, the interaction is completely wetting, and a liquid film will form on the surface when the bulk fluid is vapor. The contact angle may be calculated from

$$
\cos \theta=\frac{\Omega_{S V}^{s}-\Omega_{S L}^{s}}{\Omega_{L V}^{s}}
$$

if the surface free energies for solid-liquid, $\Omega_{S L}^{s}$, solidvapor, $\Omega_{S V}^{s}$ and liquid-vapor, $\Omega_{L V}^{s}$ can be calculated or measured.

Previous studies of wetting from a statistical mechanics point of view have focused on homogeneous surfaces. The contact angles have been predicted with both density functional theory (DFT) and molecular simulation $[16,17]$. In addition, pre-wetting transitions between a thin adsorbed layer and a thicker (but not infinite) film at a surface immersed in a vapor have been identified and characterized [18-21].

Here we discuss the effects of surface chemical heterogeneity on wetting. A similar model has recently been explored by Schoen and Diestler [22] in the context of fluid structure and surface and line tensions as well as Bock and Schoen [23] and Rocken and Tarazona [24] in the context of capillary condensation. Other theoretical

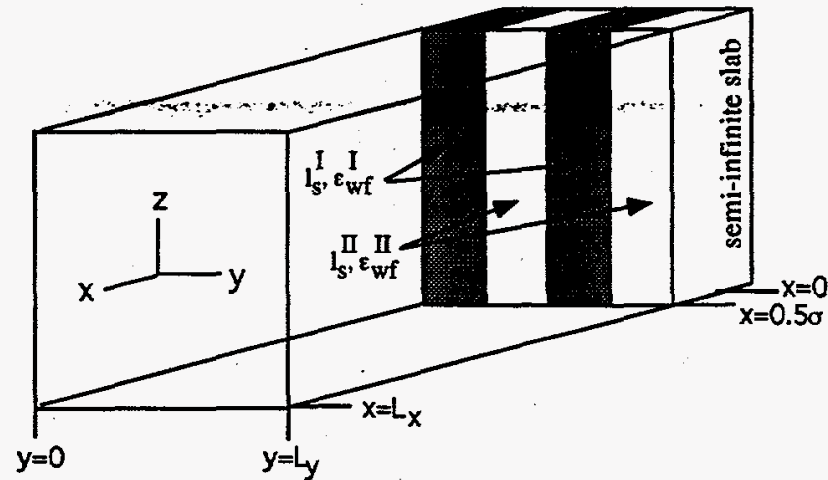

FIG. 1. A diagram of a chemically heterogeneous surface model. The surface is composed of stripes of alternating surface chemistry defined by the wall-fluid interaction potential parameters, $\epsilon_{w f}^{I}$ and $\epsilon_{w f}^{I I}$ and the extent of each stripe, $l^{I}$ and $l^{I I}$. The length of the computational domain perpendicular to the surface is $L_{x}$ and parallel to the surface is $L_{y}$. The origin of the computational domain is located inside the surface such that the fluid-surface interface is found at $x=0.5 \sigma$.

studies of chemically heterogeneous surfaces have focused on lateral wetting transitions in microscopic droplets on patterned surfaces [4], and investigations of line tensions at heterogeneous surfaces [25]. In all of these cases, bocal density functional theories have been applied. In this paper, the wetting of a heterogeneous surface is studied using a nonlocal DFT. We discuss the structure in the fluid near the inhomogeneous surface, the contact angles as calculated from Eq.1, and the phase transitions in the fluid. The results have implications for both modeling of macromolecular systems such as proteins and for tailoring materials and devices at the nanometer length scale.

\section{THEORY AND MODEL}

The heterogeneous surface model we have used is sketched in Fig.1. The planar surface is split into stripes that alternate between type I (hydrophobic) and type II (hydrophilic). Each surface type is defined by its extent, $l$, and the magnitude of the interaction energy parameter with the fluid, $\epsilon_{w f}$. Density distributions are assumed to be uniform in $z$ since the surface properties are uniform in this dimension.

The underlying molecular model describing fluid-fluid interactions is the cut and shifted 12-6 Lennard-Jones (LJ) potential, $u(r)=u_{L J}(r)-u_{L J}\left(r_{c}\right)$, where

$$
u_{L J}(r)=4 \epsilon\left[\left(\frac{\sigma}{r}\right)^{12}-\left(\frac{\sigma}{r}\right)^{6}\right]
$$

$\sigma$ is the diameter of the solvent molecule, $\epsilon$ is the energy parameter controlling the strength of fluid-fluid interactions, and $r_{\mathrm{c}}$ is the cutoff distance for the potential. The wall-fluid interactions are also cut and shifted with $v(r)=v_{L J}(r)-v_{L J}\left(r_{c, w f}\right)$, and 


$$
v_{L J}(r)=4 \epsilon_{w f}\left[\left(\frac{\sigma_{w f}}{r}\right)^{12}-\left(\frac{\sigma_{w f}}{r}\right)^{6}\right] .
$$

In Eq.3, $\sigma_{w f}$ is the characteristic interaction length of wall-fluid interactions, $\epsilon_{w f}$ is the wall-fluid energy parameter, and $r_{c, w f}$ is the cutoff for the wall-fluid interactions. The external field $V^{e x t}(\mathbf{r})$ acting on a fluid particle located at $\mathbf{r}$ due to its interactions with the heterogeneous surface is found by numerical integration over the volume of the surface, $\mathbf{R}_{S}$, via

$$
V^{e x t}(\mathbf{r})=\int d \mathbf{R}_{S} \rho_{s}\left[v_{L J}\left(\left|\mathbf{r}-\mathbf{R}_{S}\right|\right)-v_{L J}\left(r_{c, w f}\right)\right]
$$

where $\rho_{s}$ is the number density of atoms in the surface, and the integral is taken over $\left|\mathbf{r}-\mathbf{R}_{s}\right| \leq r_{c, w f}$.

Even this simple model embodies a great deal of complexity. For the case of a homogeneous surface, wetting is controlled by four field parameters: the temperature, $T, \rho_{s}, \epsilon_{w f}$, and $\sigma_{w f}$. The heterogeneous surface model presented here has eight field parameters: $T, \rho_{s}, l^{I}, l^{I I}$, $\epsilon_{w f}^{I}, \epsilon_{w f}^{I I}, \sigma_{w f}^{I}$, and $\sigma_{w f}^{I I}$. Since we will confine the discussion to a bulk fluid at liquid-vapor coexistence, the chemical potential, $\mu$ of the fluid is immediately defined by the temperature as $\mu=\mu_{\text {coex }}(T)$.

In a previous investigation of capillary condensation, Bock and Schoen [23] considered variations in $l^{I}$ (the hydrophobic part of the surface) and pore size in molecular simulations as well as $T$ and $\mu$ in a mean field lattice model while holding $\epsilon_{w f}^{I}=0.001, \epsilon_{w f}^{I I}=1.25$, and $l^{I I}=4 \sigma$.

In this paper, $\epsilon^{I I}, l^{I}$, and $l^{I I}$ are varied while the other parameters are fixed. Specifically, $k T / \epsilon=0.8$ ( $k$ is the Boltzmann constant), $p_{s} \sigma^{3}=1, \epsilon_{w f}^{I}=0.1 \epsilon$, and $\sigma_{w f}^{I}=\sigma_{w f}^{I I}=\sigma$. The cutoffs are varied from, $r_{c, w f}=r_{c}=2.5 \sigma$ to $10 \sigma$. The bulk liquid and vapor coexistence densities for the various cutoffs are $\rho_{l} \sigma^{3}=0.709$ and $\rho_{v} \sigma^{3}=0.0114$ for $r_{c}=2.5 \sigma, \rho_{l} \sigma^{3}=0.778$ and $\rho_{v} \sigma^{3}=0.0044$ for $r_{c}=5 \sigma$, and $\rho_{l} \sigma^{3}=0.785$ and $\rho_{\nu} \sigma^{3}=0.0039$ for $r_{c}=10 \sigma$.

The nonlocal DFT we apply here is the fundamental measures DFT of Rosenfeld [26]. Attractions are treated with a strict mean mean field approximation. Briefly, DFT is based on the functional minimization of the grand potential [27], $\Omega$, with respect to the fluid density distribution, $\rho(\mathbf{r})$,

$$
\frac{\delta \Omega}{\delta \rho(\mathbf{r})}=0
$$

$\Omega$ is the relevant free energy because the chemical potential, $\mu$ is constant in the interfacial region due to free material exchange with the molecules in the surrounding bulk solvent.

The grand free energy ( $\Omega=F-G$ ) is the difference between the Helmholtz free energy, $F$ and Gibbs free energy, $G=\mu N$ where $N=\int d \mathbf{r} \rho(\mathbf{r})$ is the number of molecules in the system. In an inhomogeneous fluid, the surface acts as a one body external field, $V^{e x t}$ in which the fluid density distributions equilibrate. Typically, the Helmholtz free energy, $F$, is split into ideal and excess contributions. The excess term is further split up by physical effects $\left(F_{e x}=F_{h s}+F_{a t t}\right)$ in a perturbation approach where hard spheres are the reference system. The general expression for $\Omega$ is then

$$
\Omega=F_{i d}+F_{h s}+F_{a t t}+\int d \mathbf{r} \rho(\mathbf{r})\left\{V^{e x t}(\mathbf{r})-\mu\right\} .
$$

where $F_{\text {id }}$ contains the ideal gas contributions to the free energy, $F_{h s}$ contains volume exclusion effects, and $F_{a t t}$ contains van der Waals attractions.

The various contributions to $\Omega$ in Eq. 6 are

$$
\begin{aligned}
& F_{\text {id }}=k T \int d \mathbf{r} \rho(\mathbf{r})\left\{\ln \left(\Lambda^{3} \rho(\mathbf{r})\right)-1\right\}, \\
& F_{\text {hs }}=\int d \mathbf{r} \Phi\left\{\bar{\rho}_{\gamma}(\mathbf{r})\right\}, \text { and } \\
& F_{\text {att }}=\frac{1}{2} \int d \mathbf{r} \int d \mathbf{r}^{\prime} \rho(\mathbf{r}) \rho\left(\mathbf{r}^{\prime}\right) u^{a t t}\left(\mathbf{r}, \mathbf{r}^{\prime}\right)
\end{aligned}
$$

where $\Lambda$ is the Debroglie wavelength, $\Phi$ is the free energy density of the hard sphere reference system, and $u^{a t t}$ is the attractive part of the fluid-fluid LJ interaction. Taking the Weeks, Chandler, Anderson formalism [28], $u_{L J}^{a t t}(r)=u_{L J}\left(r_{\min }\right)$ for $r \leq r_{\min }$ and $u_{L J}^{a t t}(r)=u_{L J}(r)$ otherwise, where $r_{\min }$ is located at the minimum of the LJ potential energy curve.

The functional minimization of Eq. 6 produces the Euler-Lagrange equations

$$
\begin{aligned}
0= & -\mu / k T+\ln (\rho(\mathbf{r})) \\
& +\frac{1}{k T} \int d \mathbf{r}^{\prime}\left(\sum_{\gamma} \frac{\partial \Phi}{\partial \bar{\rho}_{\gamma}} w^{(\gamma)}\left(\left|\mathbf{r}-\mathbf{r}^{\prime}\right|\right)\right) \\
& +V^{e x t} / k T+\frac{1}{k T} \int d \mathbf{r}^{\prime}\left[\rho\left(\mathbf{r}^{\prime}\right) u^{a t t}\left(\left|\mathbf{r}-\mathbf{r}^{\prime}\right|\right)\right]
\end{aligned}
$$

which are solved for the equilibrium density distrubution, $\rho(\mathbf{r})$.

The hard sphere free energy density, $\Phi$ was derived from scaled particle theory in terms of the nonlocal densities, $\bar{\rho}_{\gamma}$, by Rosenfeld [26]. These nonlocal densities are

$$
\bar{\rho}_{\gamma}(\mathbf{r})=\int d \mathbf{r}^{\prime} \rho\left(\mathbf{r}^{\prime}\right) w^{(\gamma)}\left(\left|\mathbf{r}-\mathbf{r}^{\prime}\right|\right)
$$

where $w^{(\gamma)}$ are the weight functions

$$
\begin{aligned}
w^{(3)}(r) & =\Theta(r-R) \\
w^{(2)}(r) & =4 \pi R w^{(1)}(r)=4 \pi R^{2} w^{(0)}(r)=\delta(r-R) \\
w^{(V 2)}(r) & =4 \pi R w^{(V 1)}(r)=(\mathbf{r} / r) \delta(r-R)
\end{aligned}
$$

where the $\mathbf{r}$ indicates a vector. These weight functions are based on the geometry of the fluid particles as $\Theta$ is the step function, $\delta$ is the Dirac delta function, and $R$ is the radius of a particle. Thus the integrals over 
weight functions are related to the volume, surface area, and radius of the particle. Note that $w^{V 1}$ and $w^{V 2}$ are vectors, and so the hard sphere free energy density is a sum of scalar and vector contributions, $\Phi=\Phi_{s}+\Phi_{v}$, with

$$
\begin{aligned}
& \Phi_{s}=-\bar{\rho}_{0} l n\left(1-\bar{\rho}_{3}\right)+\frac{\bar{\rho}_{1} \bar{\rho}_{2}}{1-\bar{\rho}_{3}}+\frac{1}{24 \pi} \frac{\bar{\rho}_{2}^{3}}{\left(1-\bar{\rho}_{3}\right)^{2}} \\
& \Phi_{v}=-\frac{\bar{\rho}_{V 1} \bullet \bar{\rho}_{V 2}}{1-\bar{\rho}_{3}}-\frac{1}{8 \pi} \frac{\bar{\rho}_{2}\left(\bar{\rho}_{V 2} \bullet \bar{\rho}_{V 2}\right)}{\left(1-\bar{\rho}_{3}\right)^{2}} .
\end{aligned}
$$

In solving the DFT, it is necessary to evaluate integrals for all the points in the fluid. These integral evaluations will often extend beyond the computational boundaries. For the system in Fig.1 where the equations simplify to a 2D problem in $x$ and $y$, the boundary conditions in $x$ are taken to be

$$
\rho(x, y)= \begin{cases}0 & \text { if } x<0 \\ \rho_{b} & x>L_{x}\end{cases}
$$

where the surface is assumed to be semi-infinite in the $x<0$ direction, and $\rho_{b}$ is the fluid density in the bulk. The boundary conditions in $y$ are periodic with

$$
\rho(x, y)= \begin{cases}\rho\left(x, y+L_{y}\right) & \text { if } y<0 \\ \rho\left(x, y-L_{y}\right) & y>L_{y}\end{cases}
$$

Given the equilibrium density distribution, the grand potential, $\Omega$, as well as the surface free energy,

$$
\Omega^{s}=\Omega-\Omega_{b}
$$

may be calculated immediately. The grand potential of the bulk solution is $\Omega_{b}=-p V$ where $p$ is the pressure in the bulk fluid and $V$ is the volume of the system. Eq.1 may then be used to calculate the contact angle.

In Fig.2, the variation of $\cos \theta$ with $\epsilon_{w f}$ is shown for a homogeneous surface. Complete drying is found for $\epsilon_{w f} \tilde{<} 0.1$ while complete wetting is found for $\epsilon_{w f}>2.0$. This result provides a basis of comparison for the heterogeneous surfaces.

\section{NUMERICAL DETAILS}

The solution of the Euler-Lagrange equations (Eq.8) is straightforward if the fluid density varies only in one dimension (e.g. homogeneous planar surfaces [29], cylindrical pores [30], spherical droplets [31]). In these cases the DFT may be solved with either successive substitution (Picard iterations) or Newton's method on a desktop workstation. However, the heterogeneous surface model requires a 2-dimensional(2D) solution of the DFT where the complexity of the numerical problem increases quite dramatically.

The two approaches that have been applied to 1D problems each have their advantages and disadvantages. Newton's method requires the storage of a large Jacobian matrix, but it is very stable. Solutions can often be found

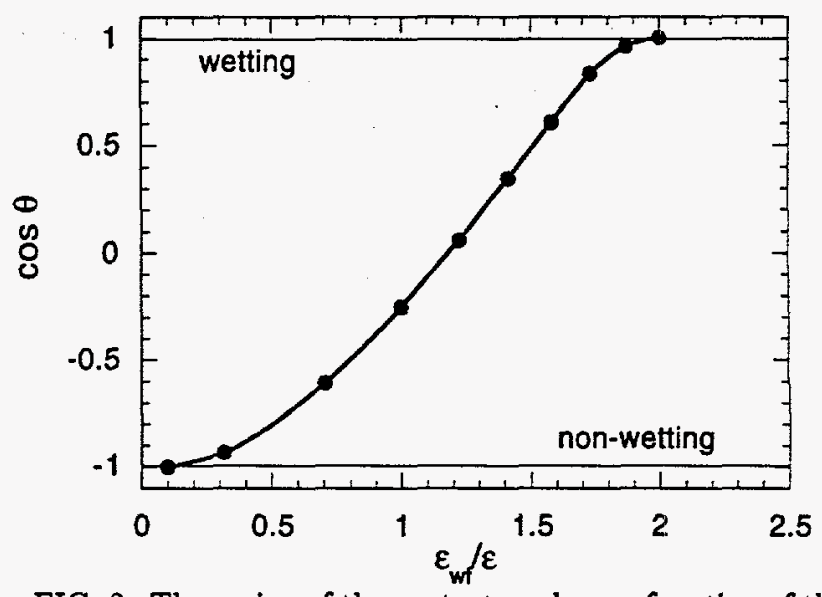

FIG. 2. The cosine of the contact angle as a function of the wall-fluid interaction potential parameter, $\epsilon_{w f}$ for a smooth homogeneous planar surface.

in $\mathrm{O}(10-20)$ Newton iterations [32]. Picard iterations are more straightforward to implement and require a great deal less memory as no Jacobian is stored; however, this approach is less stable requiring careful mixing of old and new solutions, and $\mathrm{O}(100-1000)$ iterations to convergence. We have implemented Newton's method to take advantage of its superior stability and convergence properties. The details of the numerical implementation will be presented elsewhere; here we briefly outline the fundamental numerical challenges and the approach used to overcome them.

The densities and equations are discretized using a 2D mesh in the $x-y$ plane in Fig.1. The initial guess $(k=0)$ is taken to be either $\rho_{i j}^{(k=0)}(x, y)=\rho_{b} e^{-V^{\text {ext }}(x, y) / k T}$ or a convenient continuation from an old solution.

Newton's method requires iterative solution of

$$
J_{i j}^{(k)} \Delta_{j}^{(k)}=-R_{i}^{(k)}
$$

where

$$
J_{i j}^{(k)}=\frac{\delta R_{i}^{(k)}}{\delta \rho_{j}^{(k)}}
$$

is the Jacobian, $\Delta_{j}^{(k)}=\rho_{j}^{(k+1)}-\rho_{j}^{(k)}$ is a difference array between newest approximation to the equilibrium solution at node $j$ in the mesh, $\rho_{j}^{(k+1)}$, and the kth iteration, $\rho_{j}^{(k)}$, and $R_{i}^{(k)}$ is the residual at node $i$ given by the Eq.8. The $i j$ (or $\mathbf{r}, \mathbf{r}^{\prime}$ ) element in the Jacobian matrix evaluated at $\rho^{(k)}$ is

$$
\begin{aligned}
J_{i j}= & \frac{\delta^{2} \Omega}{\delta \rho(\mathbf{r}) \delta \rho\left(\mathbf{r}^{\prime}\right)}=\frac{\delta\left(\mathbf{r}, \mathbf{r}^{\prime}\right)}{\rho(\mathbf{r})}+\frac{1}{k T} u^{a t t}\left(\mathbf{r}, \mathbf{r}^{\prime}\right) \\
& +\frac{1}{k T} \int d \mathbf{r}^{\prime \prime} \sum_{\gamma} \sum_{\xi} \frac{\partial^{2} \Phi}{\partial \bar{\rho}_{\gamma} \partial \bar{\rho}_{\xi}} w^{(\gamma)}\left(\mathbf{r}, \mathbf{r}^{\prime \prime}\right) w^{(\xi)}\left(\mathbf{r}^{\prime}, \mathbf{r}^{\prime \prime}\right)
\end{aligned}
$$

where $\delta\left(\mathbf{r}, \mathbf{r}^{\prime}\right)$ is the delta function. There are three fundamental difficulties that arise from Newton's 
method. The first is the memory needed to store the Jacobian, the second is the complexity involved in filling the Jacobian, and the third is the matrix inversion required to solve Eq.15. The memory required ultimately depens on the attractions. The range of the attractions is $2 r_{c}$. Given a typical mesh size of $\Delta=0.1 \sigma$ the number of nonzero entries in a given row of the Jacobian will be $O\left(50-200\right.$ ) (for $r_{c}=2.5-10 \sigma$ ) for a 1D solution but $O\left(2-8 \times 10^{3}\right)$ for a $2 \mathrm{D}$ problem. The total memory required for a domain that is $10 \sigma$ on an edge is then $O(40-160 k B)$ for the $1 \mathrm{D}$ problem and $O(160-640 \mathrm{MB})$ for the 2D problem.

The computational complexity of the Jacobian fill arises from the volume exclusion term in Eq.17. In order to fill this term, $\mathrm{O}\left(A \times N_{s t e n}^{2} \times N_{u n k}\right)$ operations are required where $N_{\text {sten }}$ is the number of quadrature points needed for the integral over a given weight function, $N_{u n k}$ is the total number of unknowns, and $A \approx 10$ is the number of floating point operations in the innermost loop. For the 1D problem, the number of operations required for a Jacobian fill is then $O\left(10^{5}\right)$; for the $2 \mathrm{D}$ problem it is $O\left(10^{9}\right)$.

Our numerical solution for the DFT is based on a parallel algorithm. Each processor owns a subset of the nodes in the computational domain as well as the rows of the Jacobian associated with those nodes. Eq.15 is solved using a parallel iterative solver (Aztec [33]) developed at Sandia National Laboratories (SNL). To further improve performance, a nonuniform mesh was implemented. The specific mesh we used had $\Delta=0.1 \sigma$ from the surface to $4 \sigma$ away from the surface, and a mesh of $\Delta=0.2 \sigma$ from $4-10 \sigma$ away from the surface. Most of the results presented here were obtained using $\sim 50$ processors of the ASCI Red (Sandia-Intel T-flops) parallel computer at SNL. Depending on the quality of the initial guess, solutions were obtained in 3 to 25 Newton iterations. The CPU time required for solutions with $r_{c}=2.5 \sigma$ and a domain size of $L_{x}=L_{y}=10 \sigma$ was $\mathrm{O}(100 \mathrm{sec})$.

\section{RESULTS}

\section{A. Density Distributions}

In order to calculate contact angles, the surface free energy, $\Omega^{s}$ must be calculated for solid-liquid (SL), solidvapor (SV), and liquid-vapor (LV) interfaces (see Eq.1). The LV need only be calculated once, but SL and SV profiles are needed for each value of the surface parameters. Fig.3 shows both SL and SV density distributions for the cases $l^{I I}=l^{I}=0.2 \sigma, 0.5 \sigma, 1.0 \sigma$, and $5.0 \sigma$ when $\epsilon_{w f}^{I I}=3.16 \epsilon$ and $r_{c}=2.5 \sigma$ (recall that $\epsilon_{w f}^{I}=0.1 \epsilon$ ). The length of the computational domain perpendicular to the surface is always $L_{x}=10 \sigma$. The minimum length of the domain parallel to the surface is $L_{y}=r_{c}$ with the constraint that $L_{y}$ must be an integer multiple of $l^{I}+l^{I I}$. In Fig.3A-F, $L_{y}=4 \sigma$ while in Fig.3G,H, $L_{y}=10 \sigma$.
When $l^{I I}=0.2 \sigma$ (Fig.3A,B), the density is nearly constant along the heterogeneous surface for both fluid states. When $l^{I I}=0.5 \sigma$ and $l^{I I}=1.0 \sigma$, inhomogeneous adsorption is found. The density variation parallel to the surface is oscillatory for the case $l^{I I}=0.5 \sigma$, but more complex for the case $l^{I I}=1.0 \sigma$. The density pattern in both cases repeats with a period of $l^{I}+l^{I I}$. In all of the cases with $l^{I I} \leq 1.0 \sigma$ the maximum density in the first peak of the WV distributions is $\rho \sigma^{3}<1.0$, and there is only one peak perpendicular to the surface. In contrast, a localized condensation is found when $l^{I I} / \sigma=5$ (Fig.3H). In this case, the maximum peak density in the WV profile, $\rho \sigma^{3} \approx 4$, is similar to the WL peak density in Fig.3G. In addition, the WV profile in Fig.3H has two layers of fluid perpendicular to the surface. 

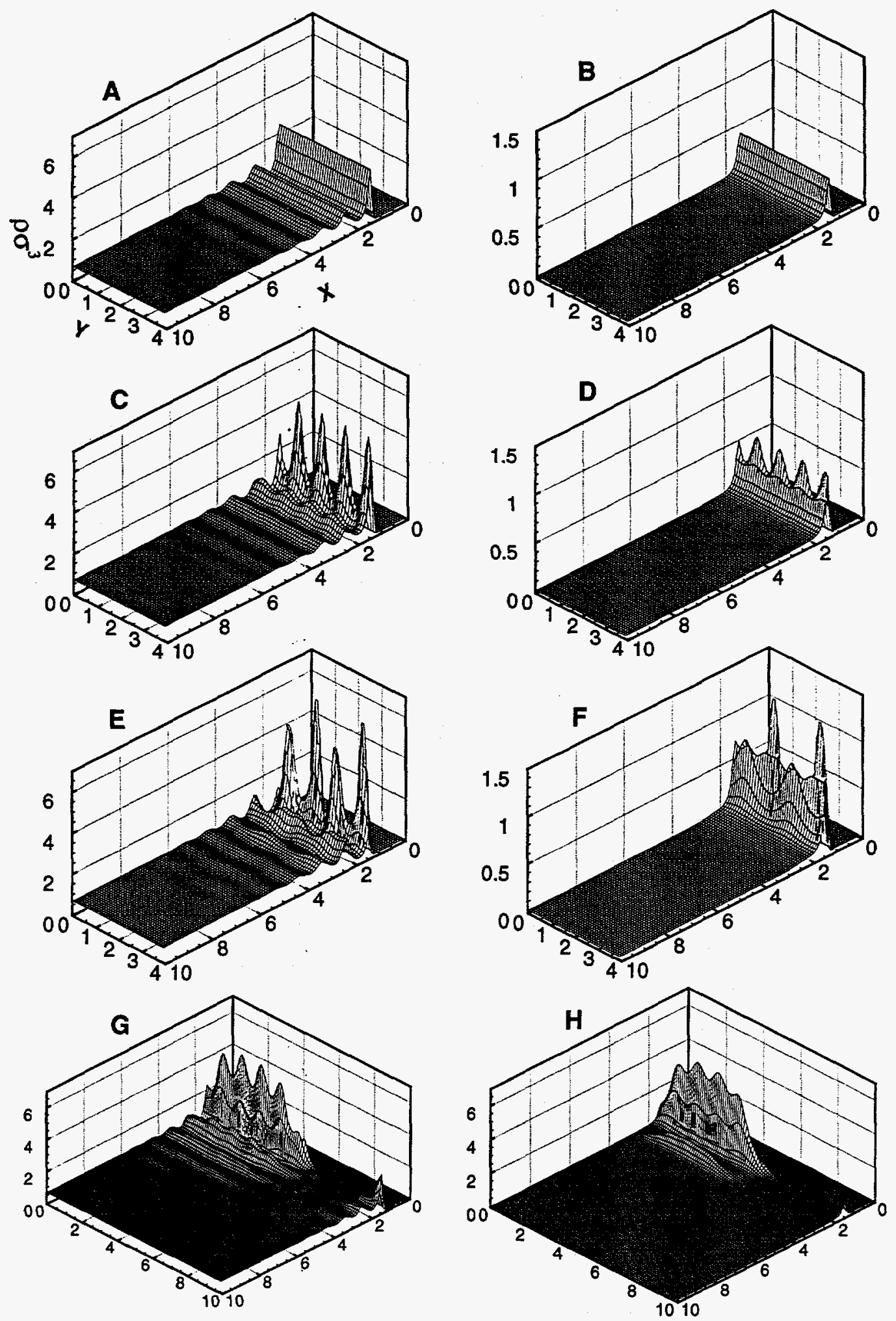

FIG. 3. Density $\left(\rho \sigma^{3}(x, y)\right)$ distributions near chemically heterogeneous surfaces with $\epsilon_{w f}^{I I}=3.16$. The different figures are distinguished by the extent of the heterogeneity (with $l^{I}=l^{I I}$ ) and the bulk fluid state as follows: $l^{I I}=0.2 \sigma(\mathrm{A}, \mathrm{B}), l^{I I}=0.5 \sigma$ $(\mathrm{C}, \mathrm{D}), l^{I I}=1.0 \sigma(\mathrm{E}, \mathrm{F})$, and $l^{H I}=5.0 \sigma(\mathrm{G}, \mathrm{H})$ with a bulk liquid in A,C,E,G, and a bulk vapor in B,D,F, and $\mathrm{H}$. The $x$ axis is perpendicular to the surfaces, and the $y$-axis is parallel to the surface. The fluid-surface interface is located at $x=0.5 \sigma$. Both $x$ and $y$ are given in units of $\sigma$. 


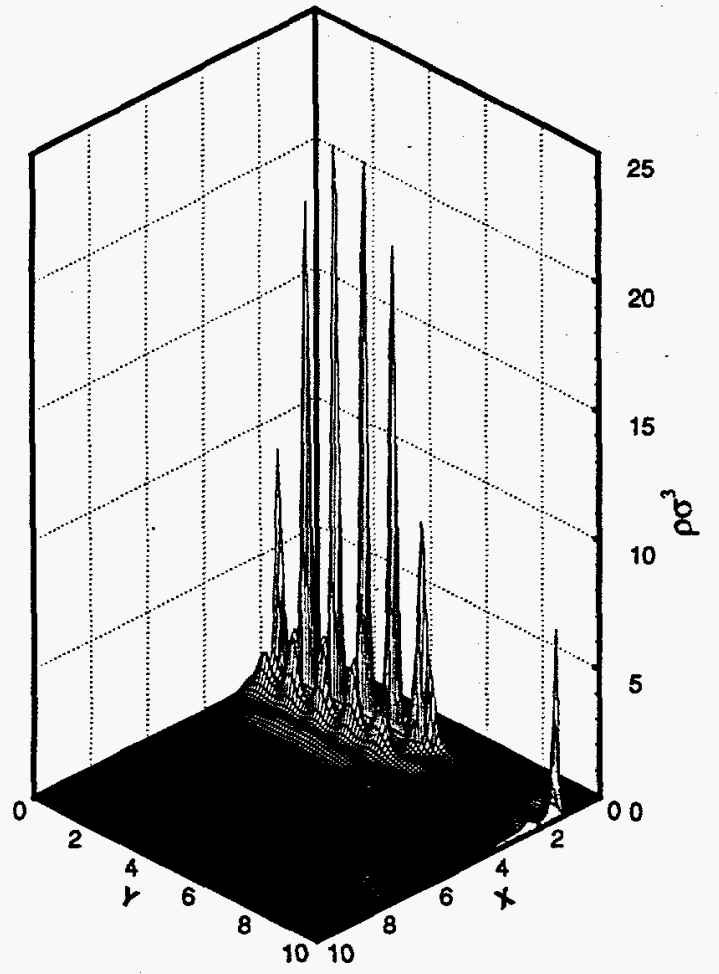

FIG. 4. The fluid density distribution, $\rho \sigma^{3}(x, y)$ near a surface with $\epsilon_{w f}^{I I}=6.32$ and $l^{I I}=5 \sigma$ when the bulk is a vapor.

The condensed state in Fig.3H is of nanoscopic extent. The liquid-vapor interface that forms between the condensed region and the surrounding vapor produces ordering parallel to the surface. There are four peaks parallel to the surface in the first layer of condensed fluid. These density oscillations have a period of $\sim 1 \sigma$.

As $\epsilon^{I I}$ is increased, the ordering within the condensed nanophase becomes more pronounced. Fig. 4 shows the density distribution corresponding to $\epsilon_{w f}^{I I}=6.32 \epsilon$ and $l^{I I}=5 \sigma$. There are now six peaks parallel to the surface in the first layer, 5 peaks in the second layer, and 4 peaks in a third layer away from the surface in the nanoscopic condensed phase. The magnitude of the peaks in the first layer is as high as $\rho \sigma^{3}=20$. For larger $\epsilon_{w f}^{I I}$, convergence becomes difficult due to the steepness of the density distributions. This numerical difficulty suggests that the system might prefer to break the symmetry in $z$ and form a nanoscopic crystal.

Nanoscopic condensed phases have been previously identified by others in studies of capillary condensation between two heterogeneous surfaces [23] In their molecular simulations, $\epsilon_{w f}^{I I}=1.25$ was small enough that ordering parallel to the surface was not observed.

The ordering parallel to the surface in Fig.4 arises from the presence of the interface between the condensed phase and the surrounding vapor. It is not an artifact of small $l^{I I}$. Fig. 5 shows the density parallel to the surface in the first peak for a case where $l^{I I}=l^{I}=30 \sigma$. Curves corre-

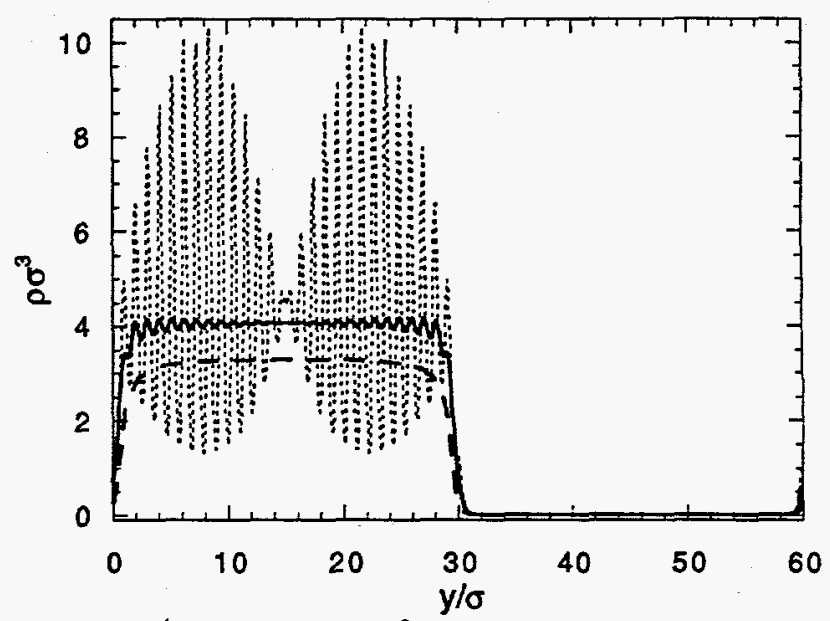

FIG. 5. The density, $\rho \sigma^{3}$ as a function of the distance parallel to the surface, $y / \sigma$, at $x=1.4 \sigma$ (recall that the fluid-surface interface is located at $x=0.5 \sigma$ ). This value of $x$ corresponds to the location of the first peak away from the surface. The three curves are: $\epsilon_{w f}^{I I}=2.24 \epsilon$ (dashed line), $\epsilon_{w f}^{I I}=2.83 \epsilon$ (solid line), and $\epsilon_{w f}^{I I}=3.16 \epsilon$ (dotted line). In all cases, $l^{I}=l^{I I}=30 \sigma$.

sponding to $\epsilon_{w f}^{I I}=2.24 \epsilon, 2.83 \epsilon$, and $3.16 \epsilon$ are included. When $\epsilon_{w f}^{I I}=2.24 \epsilon$, a constant peak density is found along much of the hydrophilic stripe $(0 \leq y / \sigma \leq 30)$. When $\epsilon_{w f}^{I I}=2.83 \epsilon$, small oscillations about the peak density are found. Finally, when $\epsilon_{w f}^{I I}=3.16 \epsilon$, a high degree of ordering is observed with peak densities of $\rho \sigma^{3} \approx 10$. Again, solutions were difficult to obtain for larger values of $\epsilon_{w f}^{I I}$.

There has been a great deal of recent interest in understanding the effects of this liquid-vapor interface, or more specifically the three phase line where the liquid-vapor interface meets the surface, on wetting and contact angles $[34,35,25,17]$. The grand potential is typically taken to be a sum of bulk $\left(\Omega_{b}\right)$, surface $(\Sigma)$, and line $(\tau)$ contributions. The surface term, $\Sigma$, is then given by the homogeneous surface limit. The presence of the three phase line in Fig.4 causes a high degree of ordering parallel to the surface which would be attributed to a large line tension. We will not pursue a lengthy discussion of line tensions here. Rather, we simply note that the line tensions would be straightforward to calculate, and emphasize that $\Omega_{s}$ as defined in Eq.14 includes all the inhomogeneities in the density profile due to both the presence of the surface and the presence of the three phase line.

\section{B. Contact Angles}

In Fig.3, $\epsilon_{w f}^{I}=0.1 \epsilon$ and $\epsilon_{w f}^{I I}=3.16 \epsilon$. A homogeneous surface with $\epsilon_{w f}=3.16 \epsilon$ would be completely wetting with $\cos \theta=1$; however a homogeneous surface with $\epsilon_{w f}=\left(\epsilon_{w f}^{I I}+\epsilon_{w f}^{I}\right) / 2=1.63 \epsilon$ would be partially wetting with $\cos \theta \approx 0.6$ (see Fig.1). For the chemically heteroge- 


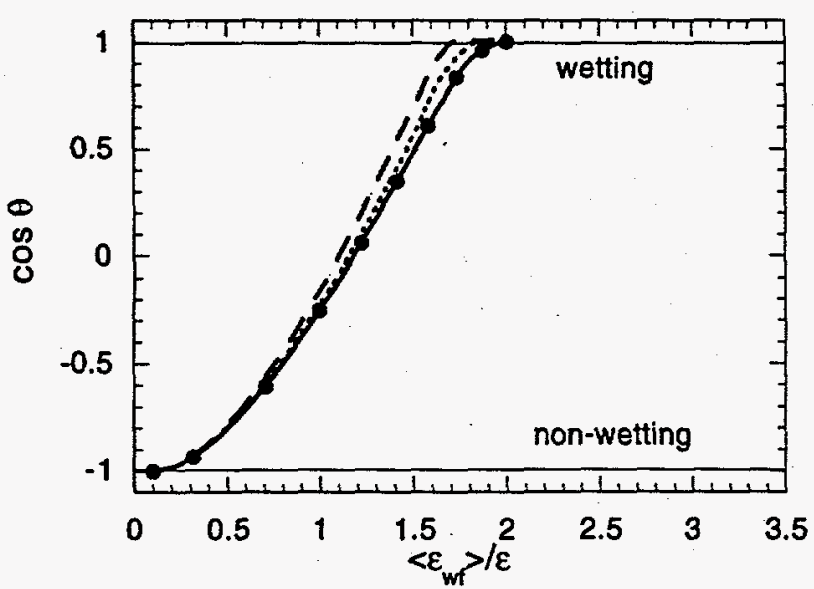

FIG. 6. The cosine of the contact angle $(\cos \theta)$ as a function of the mean wall-fluid interaction parameter, $\left\langle\epsilon_{w f}>=0.5 *\left(\epsilon_{w f}^{I}+\epsilon_{w f}^{I I}\right)\right.$, for the case $l^{I}=l^{I I}$. The various curves are: $l^{I I}=0.2 \sigma$ (solid line), $l^{I I}=0.5 \sigma$ (dotted line), and $l^{I I}=1.0 \sigma$ (dashed line). The homogeneous case (see Fig.2) is indicated by the $\bullet$.

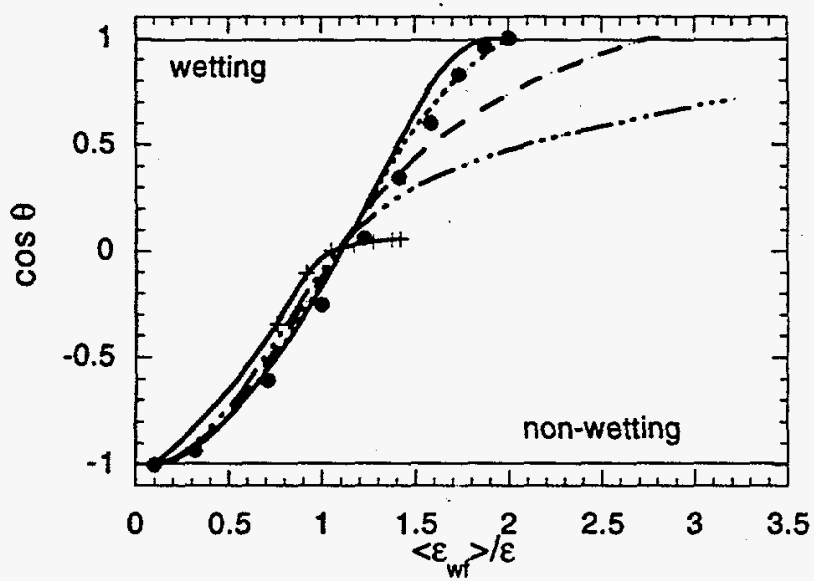

FIG. 7. See caption of Fig.6. The various curves here are: $l^{I I}=1.5 \sigma$ (solid line), $l^{I I}=2 \sigma$ (dotted line), $l^{I I}=3 \sigma$ (dashed line), $l^{I I}=5 \sigma$ (dashed-dotted line), and $l^{I I}=30 \sigma$ $(+)$. The homogeneous case (see Fig.2) is indicated by the $\bullet$.

neous surfaces, the contact angle depends on the extent of the inhomogeneity, $l^{I}$ and $l^{I I}$.

In order to systematically study wetting for the chemically heterogeneous surfaces, DFT calculations were performed for $0.2 \sigma \leq l^{I I}=l^{I} \leq 30 \sigma$ varying $\epsilon_{w f}^{I I}$ through the complete range of contact angles. The values of $\cos \theta$ were calculated from Eq.1, and the results are plotted in Figs. 6 and 7.

Fig.6 shows the variation of $\cos \theta$ with the average wallfluid interaction energy parameter for $l^{I I} \leq 1.0 \sigma$. When $l^{I I}=0.2 \sigma$, the homogeneous wall results are recovered indicating that the solvent does not distinguish heterogeneity on this length scale. When $l^{I I}=0.5 \sigma$ and $1 \sigma$, a positive deviation from the homogeneous result is observed. In these cases, a chemically heterogeneous surface is more easily wet than the homogeneous surface.

Fig.7 shows the cases $l^{I I}>1 \sigma$. The trends in Fig.6

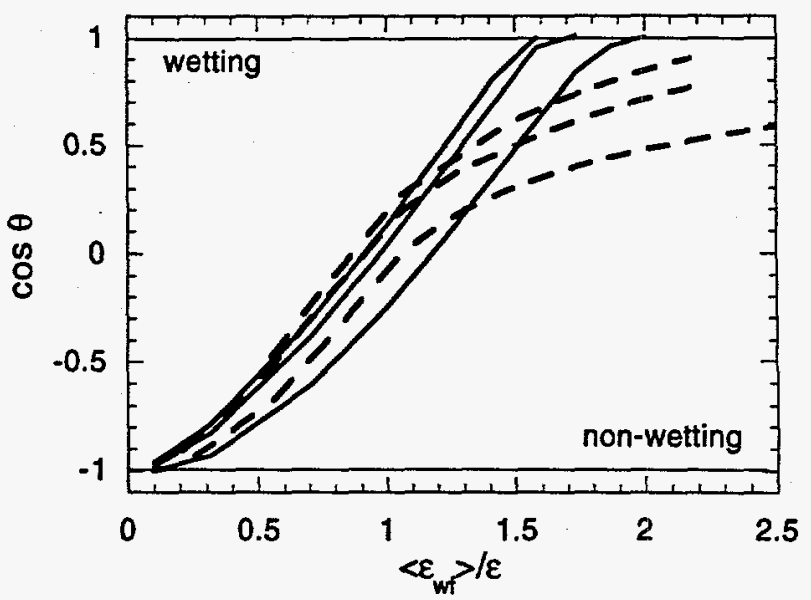

FIG. 8. See caption of Fig. 6 The various curves have different $r_{c}$. The solid lines indicate homogeneous surface results while the dashed lines correspond to heterogeneous surfaces with $l^{I}=l^{I I}=5 \sigma$. From right to left in both cases, the curves are $r_{c}=2.5 \sigma, 5 \sigma$, and $10 \sigma$.

are now reversed, and it becomes more difficult to reach complete wetting as $l^{I I}$ increases. A negative deviation from the homogeneous surface is found for $l^{I I}>2 \sigma$. For the cases $l^{I I}=5 \sigma$ and $l^{I I}=30 \sigma$, the curves stop at the points where convergence became difficult due to ordering in the nanoscopic condensed region (see Figs. 4 and 5. At these points, the $\Omega^{s}$ for the condensed nanophase and a thick wetting film were compared. In both cases, the condensed nanophase has the lower free energy.

While the location of the wetting transition depends strongly on surface chemical heterogeneity, the crossover from partial drying $(\cos \theta<0)$ to partial wetting $(\cos \theta>$ 0 ) is not very sensitive to chemical heterogeneity. For all the cases considered here, the point where $\cos \theta=0$ is found in the range $\left\langle\epsilon_{w f}\right\rangle=1.1 \pm 0.1$.

The location of the wetting transition can be expected to depend on the potential cutoffs, $r_{c}$ and $r_{c, w f}$. We have taken $r_{c}=r_{c, w f}$ as is conventional in molecular simulations of solvated macromolecular systems. An alternate approach often used when studying inhomogeneous fluids is to take $r_{c, w f}=\infty$. The dependence of the contact angle on the cutoffs is shown in Fig.8. The results for both a homogeneous surface and a heterogeneous surface $\left(l^{I I}=5 \sigma\right)$ using cutoffs of $r_{c}=r_{c, w f}=2.5 \sigma, 5 \sigma$, and $10 \sigma$ are shown. For all $r_{c}$, the suppression of complete wetting is found at the heterogeneous surface, but the effect is less pronounced for larger $r_{c}$.

\section{Phase Transitions}

Fig.7 demonstrates that the presence of chemical inhomogeneity on a surface can have a profound effect on the wetting transition where a thick liquid film forms at a solid-vapor interface (i.e. where $\cos \theta=1$ ). In this section details of both the wetting transition and other 


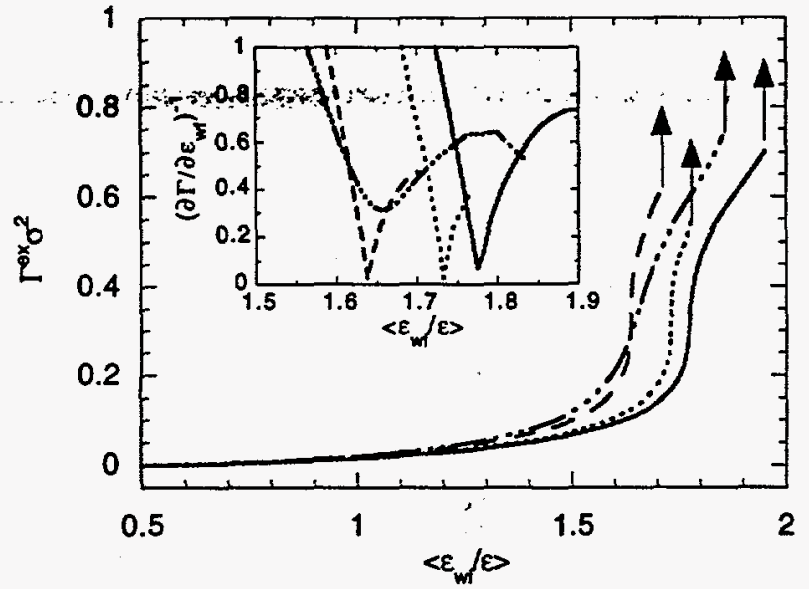

FIG. 9. The excess adsorption per unit area, $\Gamma^{e x}=\int\left[\rho(\mathbf{r})-\rho_{b}\right] d \mathbf{r}$, as a function of the mean wall-fluid interaction parameter, $\left\langle\epsilon_{w f}\right\rangle=\left(\epsilon_{w f}^{I}+\epsilon_{w f}^{I I}\right) / 2$. The various curves are: homogeneous surface (solid line), $l^{I I}=0.5 \sigma$ (dotted line) $l^{I I}=1 \sigma$ (dashed line), and $l^{I I}=1.5 \sigma$ (dashed-dotted line). The arrows indicate the location of the wetting transition. The inset shows the inverse of the slope, $\left(\partial \Gamma / \partial<\epsilon_{w f}>\right)^{-1}$ for the same $l^{I I}$ as in the main figure.

phase transitions in the fluid near the heterogeneous surface are presented. In general, first order phase transitions occur where there are discontinuities in adsorption, $\Gamma=\int \rho(\mathbf{r}) d \mathbf{r}$, and slope changes in the surface free energy, $\Omega^{s}$. Second order phase transitions occur where the slope of the adsorption is infinite but not discontinuous. First order phase transitions are typically accompanied by significant hysteresis, and so the location of the transitions was found precisely by locating the crossover of $\Omega^{8}$ for the various candidate phases.

Fig.9 shows the approach to the wetting transition for $l^{I I} \leq 1.5 \sigma$ when $l^{I}=l^{I I}$. The arrows in the figure indicate where the wetting transition to an infinite liquid film occurs. The wetting transition was found to be strongly first order in all cases with significant hysteresis of both partial wetting and thick film profiles.

For some $l^{I I}$, there may be a continuous transition occurring immediately before the wetting transition. In the inset of Fig.9, the inverse of the slope of the adsorption is plotted. While the case $l^{I I}=1.5 \sigma$ is clearly supercritical with a smoothly varying positive inverse slope, all the other cases appear near critical with a cusp-like behavior in the slope. In addition to a cusp, the inverse of the slope should reach zero at a continuous transition. This appears to be the case for both $l^{I I}=0.5 \sigma$ and $l^{I I}=1 \sigma$. This continuous transition can be identified as a prewetting transition where the prewetting critical point is on or near the bulk coexistence curve.

A phase diagram showing the effects of surface heterogeneity on the wetting transition is given in Fig.10. The figure includes results from calculations where $l^{I I} / l^{I}=$ $0.5,1$ and 2. As $l^{I I} / l^{I}$ increases, the region of complete wetting increases in size. This occurs because the sur-

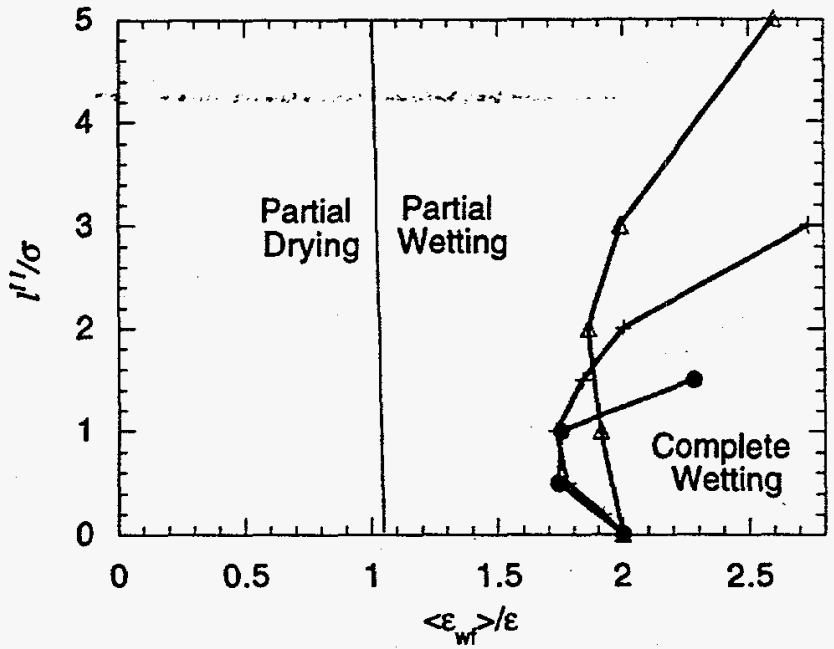

FIG. 10. A phase diagram in the $l^{I I}-<\epsilon_{w f}>$ plane where $<\epsilon_{w f}>=\left(l^{I} \epsilon_{w f}^{I}+l^{I I} \epsilon_{w f}^{I I}\right) /\left(l^{I}+l^{I I}\right)$. Regions of complete wetting $(\cos \theta=1)$, partial wetting $(0<\cos \theta<1)$ and partial drying $(-1<\cos \theta<0)$ are indicated. The line of wetting transitions is shown for the cases: $l^{I I} / l^{I}=2(\Delta), l^{I I} / l^{I}=1$ $(+)$, and $l^{I I} / l^{I}=0.5(\bullet)$.

face is becoming increasingly dominated by hydrohilic effects. In all cases, the complete wetting region cannot be reached at all if $l^{I}$ is sufficiently large $\left(l^{I} \approx 3 \sigma\right)$ regardless of the value of $\epsilon_{w f}^{I I}$.

In addition to the global wetting transition, the appearance of the nanoscopic condensed state in Fig. $3 \mathrm{H}$, and its significant effect on the contact angle (Fig.7) naturally lead to the question of whether any new phase transitions arise as a result of surface inhomogeneity. In Bock and Schoen's investigation of capillary condensation, a first order transition to a nanoscopic bridge phase occurred when the fluid was confined between two chemically heterogeneous surfaces [23].

Any new phase transitions with respect to wetting would be expected to occur where the nanoscopic condensed phase appears (ie. for $l^{I I} \geq 3 \sigma$ when $l^{I I}=l^{I}$ ). The adsorption curves for these cases are shown in Fig.11. No indications of a first order transition (e.g. hysteresis) could be found for $l^{I I} \leq 10 \sigma$. One first order transition was found when $l^{I I}=20 \sigma$ and two were located for the case $l^{I I}=30 \sigma$. They occur at the discontinuous jumps indicated by the arrows in Fig.11.

The transitions in Fig.11 are local layering transitions. At each jump in adsorption, one layer of fluid in the nanoscopic condensed region is becoming liquidlike. Fig.12 shows the density profiles perpendicular to the surface at the center of the hydrophilic $(y=15 \sigma)$ region of the $l^{I}=l^{I I}=30 \sigma$ surface for $\left\langle\epsilon_{w} f\right\rangle$ $/ \epsilon=1.04,1.05,1.10$, and 1.11. These four vaules of $<\epsilon_{w} f>/ \epsilon$ correspond to points just below and above the two transitions. They correspond to a transition from gas-like to liquid-like behavior in the second and third layers away from the surface respectively.

The local layering transition is first order in that the 


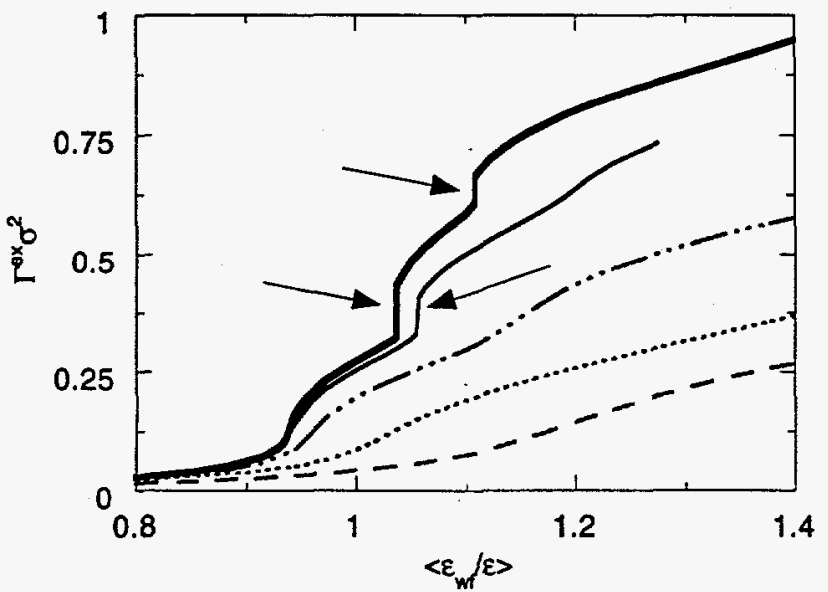

FIG. 11. See caption of Fig.9. The various curves here are: $l^{I I}=3 \sigma$ (dashed), $l^{I I}=5 \sigma$ (dotted), $l^{I I}=10 \sigma$ (dashed-dotted), $l^{I I}=20 \sigma$ (light solid), and $l^{I I}=30 \sigma$ (heavy solid). In all cases $l^{I I} / l^{I}=1$.

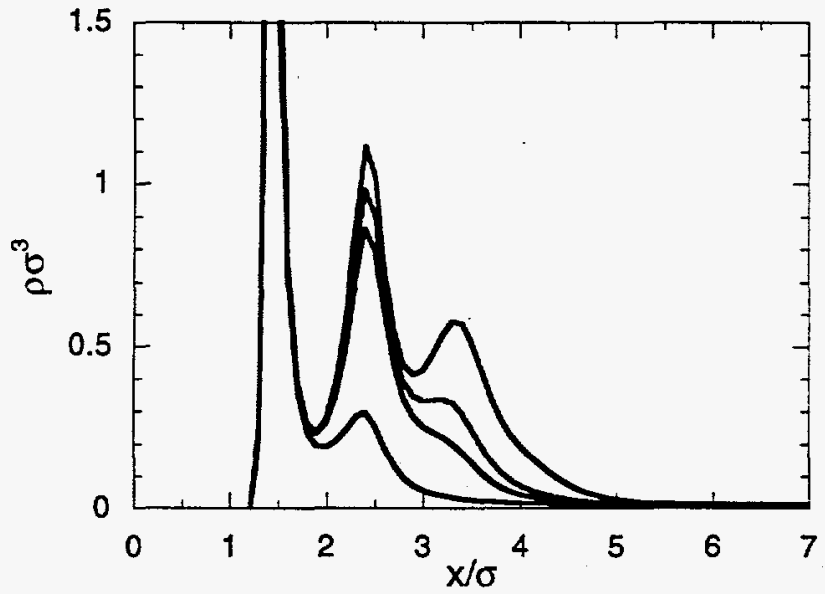

FIG. 12. The density, $\rho \sigma^{3}$ perpendicular to the surface at the location $y=15 \sigma$ (see Fig.5). The various cuves correspond to different values of $\epsilon_{w f}^{I I}$. From left to right: $\left.\left.\epsilon_{w f}^{I I}=1.97 \epsilon\left(<\epsilon_{w f}\right\rangle=1.04\right), \epsilon_{w f}^{I I}=2.00 \epsilon\left(<\epsilon_{w f}\right\rangle=1.05\right)$, $\left.\left.\epsilon_{w f}^{I I}=2.10 \epsilon\left(<\epsilon_{w f}\right\rangle=1.10\right), \epsilon_{w f}^{I I}=2.12 \epsilon\left(<\epsilon_{w f}\right\rangle=1.11\right)$,

adsorption increases discontinuously and hysteresis of states is observed near the transition. It is weakly first order in that the hysteresis is not extensive, and the change in slope in $\Omega^{s}$ is quite small. The local layering transition and its first order nature are related to the wetting transition of a homogeneous surface. This is apparent from the fact that the local layering transitions occur in the vicinity of $\epsilon_{w f}^{I I}=2 \epsilon\left(\left\langle\epsilon_{w f}\right\rangle=1 \epsilon\right)$ where wetting transition would occur for a homogeneous surface.

A phase diagram showing the local layering transitions, the global wetting transition, and the lines of numerical instability due to ordering in the nanoscopic condensed region for the case $l^{I I}=l^{I}$ is shown in Fig.13. There are two lines of numerical instability for the wall-liquid profiles. The upper curve is related to a local ordering near the hydrophilic parts of the surface. The lower curve is related to ordering along the entire surface. While the

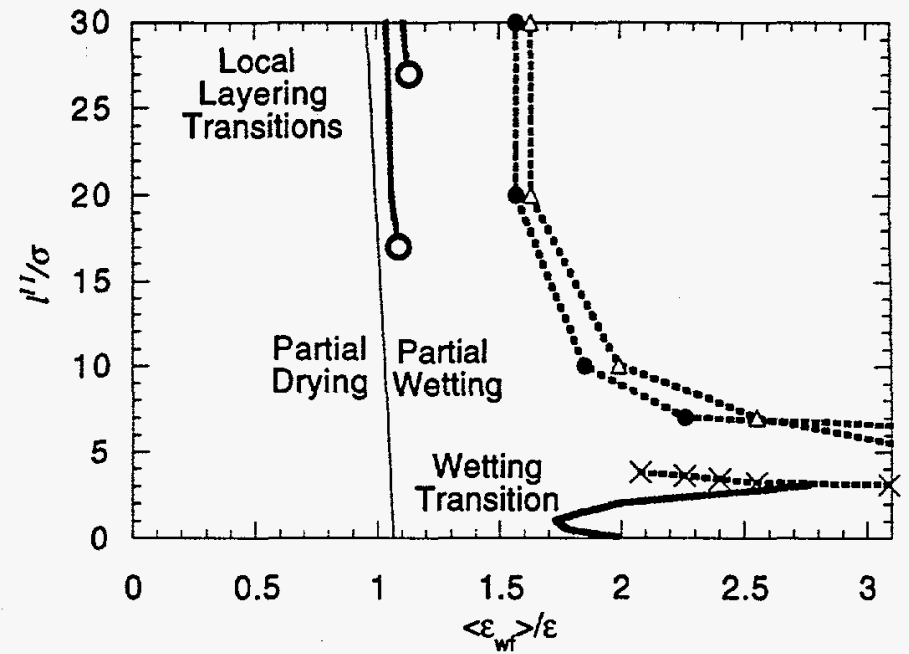

FIG. 13. A phase diagram in the $l^{I I}-<\epsilon_{w f}>$ plane for the case $l^{I I}=l^{I}$. The solid lines indicate local layering transitions and the global wetting transition. The critical endpoints of the layering transitions ( 0 ) were estimated from adsorption curves (e.g. Fig.11). The dashed lines indicate numerical instabilities that arise from ordering in the fluid. The various curves are: instabilities in the WV profiles $(\Delta)$, WL profiles (• and $x$ ). The WL profiles given by $\bullet$ correspond to nanoscopic ordering while the $x$ correspond to global ordering.

structure in the fluid near these points of numerical instability suggest that crystallization might occur, verification with further calculations is needed. Furthermore precise location of the transition would require calculation of the free energies of the solid phases.

\section{CONCLUSIONS}

In this paper, we have presented the results of nonlocal 2D DFT calculations on the wetting properties of chemically heterogeneous surfaces. These calculations showed complex density distributions and phase behavior as a result of the heterogeneity. Condensed phases of nanoscopic extent were obtained and found in many cases to be very stable as compared with thick wetting films. The condensed nanophase is in the most general sense a manifestation of partial wetting. However, these phases are far from simple leading to the local layering transitions discussed here as well as the bridge phases discussed by others [23].

The location of the wetting transition was found to be strongly dependent on the nature (extent and strength) of the heterogeneity, and complete wetting was suppressed altogether if the hydrophobic parts of the surface were large enough. In these cases, the condensed nanophase may crystallize if the hydrophilic surface-fluid interactions are strong enough. Such nanoscopic crystals could act as nucleation sites for the growth of a bulk crystal. Further 3D DFT or molecular simulations are needed to verify the existence and nature of a nanoscopic 
crystalline phase.

The results in this paper have implications for models of self-assembly and biological systems. Many models of these systems assume that self-assembly (e.g. protein folding) may be understood with simple two state (hydrophobic/hydrophilic) models: This is likely to be the case when the extent of the different surface types are large enough to support significant differences in local solvent density. The calculations here suggest that this requires the extent of the inhomogeneities to be at least $3-4 \sigma$ or about $1 \mathrm{~nm}$.

Finally, the phase diagram in Fig.13 can be viewed as an operational phase diagram for designing nanoscopically tailored devices and materials [36]. It shows where highly ordered and perhaps crystalline nanoscopic phases can be obtained. It also shows that the lower bound on the extent of the hydrophobic part of the surface for formation of distinct nanoscopic phase is $\sim 4 \sigma$. If the hydrophobic portions of the surface are smaller than this limit, the adsorbate will form thick wetting films rather than nanoscopically ordered phases.

Future work should address in more detail the possibility of nanoscopic crystallization. In addition, lateral phase transitions may occur as the condensed phase expands to form a wetting film. Lenz and Lipowsky have shown that such lateral transitions may result in microscopic fluid domains that span several of the underlying hydrophobic and hydrophilic stripes [4]. In order to observe these structures, the computational domain parallel to the surface will need to be extended to include many repetitions of the surface stripes.

\section{ACKNOWLEDGEMENTS}

Sandia is a multiprogram laboratory operated by Sandia Corporation, a Lockheed Martin Company, for the United States Department of Energy under Contract DEAC04-94AL85000.

[1] D.W.L. Tolfree, Rep. Prog. Phys., 61, 313 (1998).

[2] J. Samuel, C.J. Brinker, L.J. Douglas Frink, and F. van Swol, Langmuir, 142602 (1998).

[3] L.J. Douglas Frink and F. van Swol, J. Chem. Phys., 108 5588 (1998).

[4] P. Lenz and R. Lipowsky, Phys. Rev. Lett. 801920 (1998).

[5] G. Gompper and S. Klein, J. de Physique II France, 2, 1725 (1992).

[6] G. Gompper and M. Kraus , Phys. Rev. E, 47, 4289 (1993).; 47, 4301 (1993).

[7] M.W. Deem and D. Chandler, Phys. Rev. E, 49, 4268 (1994).
[8] K.A. Dill, Biochemistry, 24, 1501 (1985).

[9] W.E. Hart and S.Istrail, J. Comp. Bio., 3, 53 (1996); 4, 241 (1997).

[10] A. Irback, C. Peterson, F. Potthast, and O. Sommelius, J. Chem. Phys., 107, 273 (1997).

[11] A. Angelova, Thin Solid Films, 243394 (1994).

[12] J.E. Seebergh and J.C. Berg, Chem. Eng. Sci., 474455 (1992).

[13] F. van Swol and J.R. Henderson, J. Chem. Soc. Faraday Trans. 2, 2, 1685 (1986).

[14] E.M. Blokhuis, Y. Shilkrot, and B. Widom, Mol. Phys., 86, 891 (1995).

[15] A.E. van Giessen, D.J. Bukman, and B. Widom, J. Colloid and Int. Sci., 192257 (1997).

[16] F. van Swol and J.R. Henderson, Phys. Rev. A, 40, 2567 (1989); 43, 2932 (1991).

[17] T. Getta and S. Dietrich, Phys. Rev. E, 57, 655 (1998).

[18] J.W. Cahn, J. Chem. Phys., 66, 3667 (1977).

[19] C. Ebner and W.F. Saam, Phys. Rev. Lett., 38, 1486 (1978).

[20] E. Velasco and P. Tarazona, Phys. Rev. A, 42, 2454 (1990).

[21] J.E. Finn and P.A. Monson, Phys. Rev. A 396402 (1989).

[22] M. Schoen and D.J. Diestler, Phys. Rev. E, 564427 (1997).

[23] H. Bock and M. Schoen, submitted to Phys. Rev. E (1998).

[24] P. Rocken, and P. Tarazona, J. Chem. Phys. 105, 2043 (1996).

[25] W. Koch, S. Dietrich, and M. Napiorkowsi, Phys. Rev. E, 51, 3300 (1995).

[26] Y. Rosenfeld, Phys. Rev. Lett., 63, 980 (1989).

[27] D. Henderson, ed., Fundamentals of Inhomogeneous Fluids, Marcel Dekker, New York (1992).

[28] J.D. Weeks, D. Chandler, and H.C. Anderson, J. Chem. Phys., 54, 5237, (1971).

[29] T.K. Vanderlick, L.E. Scriven, and H.T. Davis, J. Chem. Phys., 00, 2422 (1989).

[30] U. Marini Bettolo Marconi and F. van Swol, Mol. Phys., 51081 (1991).

[31] V. Talanquer and D.W. Oxtoby, J. Chem. Phys., 100, 5190 (1994).

[32] J.R. Henderson and Z.A. Sabeur, J. Chem. Phys., 97, 6750 (1992).

[33] S.A. Hutchinson, L.V. Prevost, R.S. Tuminaro, and J.N. Shadid. Aztec user's guide: Version 2.0. Technical report, Sandia National Laboratories, Albuquerque, NM 1998.

[34] B. Widom and H. Widom, Physica A, 173, 72 (1991).

[35] B. Widom, J. Phys. Chem., 99, 2803 (1995).

[36] L. Yan, X.M. Zhao, and G.M. Whitesides, J. Amer. Chem. Soc., 1206179 (1998). 